\title{
Adaptação na Transição para a Escola: Estudo do Questionário de Adaptação Escolar e da Escala de Competência Social do SSBS-2
}

\author{
Adaptation in the Transition to School: School Adaptation Questionnaire and Social \\ Competence Scale SSBS-2
}

\author{
Karla Correia $^{1}$, Alexandra Marques-Pinto ${ }^{2}$ e Magda Sofia Roberto ${ }^{3}$
}

\begin{abstract}
Resumo
As qualidades psicométricas de dois instrumentos - o Questionário de Adaptação Escolar, versões para crianças e para professores, e a versão portuguesa da escala de Competência Social do School Social Behavior Scales (SSBS-2) - foram analisadas numa amostra de 13 educadores e 14 professores e num total de 328 crianças que frequentavam o último ano da educação pré-escolar e o primeiro ano de escolaridade. Os resultados salientam uma estrutura em dois fatores com boa consistência interna, fidedignidade teste / reteste e validade de constructo da versão para professores do Questionário de Adaptação Escolar, uma estrutura unifatorial, boa consistência interna mas um índice de estabilidade temporal mais fraco da versão para crianças, e validade fatorial e fidedignidade da escala de Competência Social do SSBS-2. Observou-se a invariância dos modelos em relação ao sexo e nível de escolaridade dos participantes permitindo a realização de análises multi-grupos.
\end{abstract}

Palavras-chave: adaptação, transição escolar, competência social, pré-escolar, primeiro ciclo

\begin{abstract}
The psychometric properties of two instruments - the School Adaptation Questionnaire, children and teacher's versions, and the Portuguese version of the Social Competence Scale of the School Social Behavior Scales (SSBS-2) - were analyzed in a sample of 13 preschool teachers, 14 primary school teachers and a total of 328 children attending the last year of pre-school and first grade. The results highlight a structure on two factors with good internal consistency, reliability test - re-test, and construct validity of the teacher's version of the School Adaptation Questionnaire, a one-factor structure, good internal consistency but a weaker temporal stability index of the children's version, and factorial validity and reliability of the Social Competence Scale of SSBS-2. Invariance was checked regarding sex and educational level, allowing for multi-group analysis.
\end{abstract}

Keywords: adaptation, transition to school, social competence, preschool, primary school

Esta investigação foi realizada no âmbito do Programa de Doutoramento em Psicologia Educacional, e foi apoiada pela Fundação para a Ciência e Tecnologia (FCT) de Portugal através da Bolsa de Doutoramento com a referência SFRH / BD / 36012 / 2007.

1 Doutoramento em Psicologia da Educação. Faculdade de Psicologia da Universidade de Lisboa. Alameda da Universidade 1649-013 Lisboa, Portugal. Tel.: 00351962465657. E-mail: correia.karla@gmail.com

${ }^{2}$ Doutoramento em Psicologia Educacional, Professora Auxiliar da Faculdade de Psicologia da Universidade de Lisboa. Alameda da Universidade 1649-013 Lisboa, Portugal. E-mail: ampinto@ @sicologia.ulisboa.pt

${ }^{3}$ Doutoramento em Psicologia Social, Professora Auxiliar Convidada da Faculdade de Psicologia da Universidade de Lisboa. Alameda da Universidade 1649-013 Lisboa, Portugal. E-mail: msroberto@psicologia.ulisboa.pt 


\section{Introdução}

O sucesso na adaptação da criança à transição escolar parece ter efeitos a longo termo nos seus resultados académicos e sociais e uma influência significativa no seu desenvolvimento (eg. Alexander, Entwisle, \& Dauber, 1993; Margetts, 2009). Existem efetivamente algumas evidências empíricas que sugerem que o ajustamento da criança ao nível académico, social e comportamental ao longo do primeiro ciclo pode ser previsto pela sua adaptação no primeiro ano de escolaridade (eg. Hamre \& Pianta, 2001; Margetts, 2009). Neste sentido, torna-se essencial identificar e intervir precocemente em situações onde existam indícios de uma adaptação escolar menos positiva. O presente estudo pretende estudar as qualidades psicométricas de dois instrumentos, o Questionário de Adaptação Escolar, versão para professores e versão para crianças, tendo em vista a avaliação da qualidade da adaptação escolar na transição para a escola; e a escala de Competência Social do School Social Behavior Scales (SSBS-2; Merrell, 2002; adaptação portuguesa de Raimundo et al., 2012), que avalia competências e traços que são caraterísticos de alunos bem adaptados e competentes do ponto de vista social.

\section{Adaptação à Escola na Transição}

A adaptação à escola pode ser conceptualizada como uma tarefa multifacetada que envolve um reajustamento às novas exigências intelectuais, sócio-emocionais e comportamentais da sala de aula, e que se reflete na aquisição de competências nestes domínios (Perry \& Weinstein, 1998). Quando a criança entra na escola é esperado que se adapte ao professor e às exigências da sala de aula, que se envolva numa série de novas interações com os pares, e que se desenvolva nos planos da autonomia e da construção de uma identidade própria (McIntyre, Blacher, \& Baker, 2006).

A forma como a adaptação à escola é definida varia consideravelmente nos diversos estudos que se lhe têm dedicado, com reflexo nas dimensões da adaptação incluídas nas medidas de avaliação propostas. Tem sido conceptualizada com base em uma ou mais dimensões: domínio académico (eg. competências ou aptidões desenvolvidas e motivação escolar), domínio social (eg. qualidade da relação com os pares) e domínio comportamental (eg. capacidade de regular o comportamento) (Perry \& Weinstein, 1998). A diversidade de definições da adaptação escolar patente nos indicadores de medida que a operacionalizam é reveladora da extensão de competências que se espera que as crianças adquiram ou desenvolvam na transição para a escola.

McIntyre, Blacher e Baker (2006) definiram como indicadores de adaptação na transição ter poucos problemas de comportamento relatados por professores e uma relação professor-aluno positiva, num estudo que procurou avaliar se as competências de autorregulação e as competências sociais das crianças previam uma adaptação positiva na transição para a escola. Serdal (2010), por seu lado, desenvolveu o questionário Adaptation to School in the First Month Questionnaire para avaliar a adaptação social e comportamental no primeiro mês de escola das crianças do primeiro ano de escolaridade, cujos itens, relativos ao cumprimento de regras, ao relacionamento com os pares e o professor, e ao interesse pela escola foram formulados para traduzir uma adaptação negativa. Akçinar (2013) num estudo sobre os preditores da adaptação escolar de crianças com seis anos de idade na Turquia utilizou a versão para pais do School Adaptation Questionnaire criado pela equipa da Early Childhood Developmental Ecologies in Turkey (Baydar, Küntay, Gökşen, Yağmurlu, \& Cemalcılar, 2007), cujos itens traduziam comportamentos indiciadores de adaptação (e.g. "o meu filho tem dificuldade em obedecer às regras da sala de aula"; "o meu filho prepara a sua mochila para a escola") a serem avaliados pelas mães. Birch e Ladd (1997) no seu estudo sobre a relação criança-professor e a adaptação escolar de crianças do jardim-de-infância utilizaram vários índices, a saber um teste de prontidão (Metropolitan Readiness Tests, Nurss \& McGauvran, 1986) que avaliava a aptidão visual e linguística das crianças; um questionário dirigido às crianças que avaliava a insatisfação social e a solidão (Loneliness and Social Dissatisfaction Questionnaire for Young Children, Cassidy \& Asher, 1992); uma escala de autorrelato de 
atitudes em relação à escola (School Liking and School Avoidance Scale, Ladd \& Price, 1987); e uma escala para professores contemplando vários aspetos associados à adaptação das crianças à escola (Teacher Rating Scale of School Adjustment, desenhada pelos autores do estudo), designadamente gosto pela escola, evitamento escolar, participação cooperativa, e autocontrolo.

No contexto português, não foram encontrados instrumentos específicos que avaliem a adaptação na transição para a escola, tendo-se identificado apenas um estudo de adaptação do questionário de Adaptação Psicossocial da Criança (APSE Questionnaire, Strayer \& Noel, 1990; adaptação de Silva, Veríssimo, \& Santos, 2004), realizado com crianças dos três aos cinco anos de idade. Os itens deste questionário avaliam comportamentos no plano escolar (linguagem, motricidade fina, etc.), psicológico (humor, empatia, etc.), comportamental (agressividade, motricidade geral) e social (popularidade, sociabilidade).

\section{Competência Social e Emocional}

A aprendizagem e o desenvolvimento de competências sociais e emocionais têm sido considerados, como vimos, indicadores relevantes da adaptação à transição escolar e estão associados à criação de relações interpessoais positivas (Consortium on the School-Based Promotion of Social Competence, 1996; Gresham \& Elliott, 1990; Lemos \& Meneses, 2002), ao sucesso académico (Gresham \& Elliott, 1990; Elias \& Weissberg, 2000; Lane, Menzies, Barton-Arwood, Doukas, \& Munton, 2005; Zins \& Elias, 2006), e ao bem-estar psicológico (Holopainen, Lappalainen, Junttila, \& Savolainen, 2012). A sua avaliação e promoção, designadamente na transição escolar, têm desta forma vindo a ser reconhecidas como aspetos fundamentais para o sucesso académico e na vida (Denham, 2006; Hemmeter, Ostrosky, \& Fox, 2006; Zins \& Elias, 2006).

A aprendizagem social e emocional (Social Emotional Learning - SEL) é o processo de aquisição e aplicação de conhecimentos, atitudes e habilidades necessárias para reconhecer e gerir as emoções; desenvolver carinho e preocupação pelos outros; tomar decisões responsáveis; estabelecer relações positivas; e lidar com Revista Iberoamericana de Diagnóstico y Evaluación - e Avaliação Psicológica. RIDEP · Nº45 · Vol.3 · 17-31 · 2017 situações desafiadoras de forma competente (Collaborative for Academic, Social, and Emotional Learning, 2003; Zins \&Elias, 2006).

Em Portugal tem existido uma crescente preocupação com a avaliação das competências sociais e emocionais, ainda que existam poucos estudos psicométricos dos instrumentos utilizados (Raimundo et al., 2012). Dos trabalhos até agora publicados são de referir os estudos de adaptação para a língua portuguesa do Preschool and Kindergarten Behavior Scales - 2 (PKBS-2, Merrell, 2002) com crianças de três a seis anos de idade (Major, 2011; Major \& Seabra-Santos, 2014a); do Affect Knowledge Test (Denham, 1986) com crianças desta mesma faixa etária (Machado, Veríssimo, \& Denham, 2012); do Social Skills Rating System, forma para professores de alunos do $1^{\circ}$ ao $6^{\circ}$ ano de escolaridade (Lemos \& Meneses, 2002) e formato de autoavaliação para alunos do $7^{\circ}$ ao $9^{\circ}$ ano (Pedro \& Albuquerque, 2007); e do School Social Behavior Scales, para avaliação pelos professores de alunos do $1^{\circ}$ ao $12^{\circ}$ ano (Raimundo et al., 2012). Apesar das boas características psicométricas evidenciadas por estas medidas em estudos com amostras da população portuguesa, nenhuma foi estudada simultaneamente com educadores de alunos do pré-escolar e professores de alunos do primeiro ano do básico, não se encontrando desta forma uma medida validada para a avaliação das competências sociais e emocionais dos alunos antes e depois da transição escolar.

Considerando a escassez de investigação no âmbito da avaliação da adaptação escolar e das competências sociais e emocionais na transição para a escola em Portugal, o presente estudo tem por objetivos construir um Questionário de Adaptação Escolar, com duas versões uma de heteroavaliação pelos professores e outra de autoavaliação pelas crianças, e analisar alguns dos seus parâmetros de validade e fiabilidade bem como da Escala de Competência Social do School Social Behavior Scales (SSBS-2; Merrell, 2002; adaptação portuguesa de Raimundo et al., 2012) com educadores e professores de crianças dos quatro aos sete anos de idade. 


\section{Método}

\section{Participantes}

A amostra de professores foi constituída por 13 educadoras e 14 professores de primeiro ciclo, maioritariamente do sexo feminino $(85,7 \%)$, de quatro jardim-de-infância e quatro escolas públicas de primeiro ciclo, respetivamente, da região de Lisboa. Estes educadores e professores completaram um total de 328 questionários, um por aluno, de 160 raparigas e 168 rapazes, entre os quatro e os sete anos de idade $(\mathrm{M}=5.50 ; \mathrm{SD}=.63)$, que frequentavam o último ano da educação préescolar $(60,6 \%)$ e o primeiro ano de escolaridade $(39,4 \%)$, respetivamente. As crianças da amostra $(\mathrm{N}=328)$, provinham maioritariamente de famílias caucasianas de nacionalidade portuguesa $(82 \%)$, sendo de assinalar a presença de crianças provenientes de minorias étnicas, nomeadamente, de famílias oriundas dos PALOP $(9,7 \%)$, da Europa do Leste (1,2\%), Ásia (1,5\%), e de etnia cigana $(5,5 \%)$. Uma subamostra composta por sete educadoras e seis professores, na sua totalidade do sexo feminino, efetuaram uma reavaliação sete meses após a primeira avaliação, de 176 crianças, com igual número de raparigas e rapazes, a frequentarem, respetivamente, o nível pré-escolar $(43,2 \%)$ e o primeiro ano de escolaridade $(56,8 \%)$.

\section{Instrumentos}

Questionário de Adaptação Escolar - versão para professores. Este instrumento foi construído a partir de entrevistas de focus group, realizadas com educadoras de jardim-de-infância $(\mathrm{N}=18)$ e professores de primeiro ciclo $(\mathrm{N}=13)$ num estudo prévio (Correia \& Marques-Pinto, 2016) que envolveu quatro agrupamentos de escola de Lisboa, em que se aferiu quais os sinais que, na perspetiva dos participantes, indiciavam uma boa adaptação escolar. Após a listagem de sinais/comportamentos indicados pelos participantes dos focus group, os itens redundantes foram eliminados e a listagem final foi apresentada a três educadores e três professores que participaram no estudo de forma a assegurar a compreensibilidade dos itens retidos. O questionário assim construído é constituído por oito itens (eg. "mostra vontade em aprender coisas novas"; "cumpre com as regras da escola e da turma") que contêm sinais de adaptação à escola, a serem avaliados pelos professores numa escala de cinco pontos (de 1=nunca a 5=sempre). Uma pontuação mais elevada indica uma melhor adaptação escolar.

Questionário de Adaptação Escolar - versão para crianças. Para a construção deste instrumento foram considerados os indicadores recolhidos nas entrevistas de focus-group com educadores e professores de primeiro ciclo, anteriormente referidas, bem como os identificados em entrevistas de focus-group com pais e crianças, antes $(\mathrm{N}=14$ e $\mathrm{N}=22$, respetivamente) e após $(\mathrm{N}=20$ e $\quad \mathrm{N}=45$, respetivamente) a transição para a escola, igualmente realizadas num estudo prévio (Correia $\&$ Marques-Pinto, 2016, 2017). Foi elaborada uma listagem de todos os indicadores recolhidos e eliminados os que continham conteúdos equivalentes, mantendo sempre que possível os itens formulados pelas próprias crianças e simplificando a linguagem dos resultantes das perspetivas de pais, educadores e professores. Da listagem final constam 9 itens (eg. "fica feliz quando vem à escola"; "Gosta de aprender coisas novas") a serem avaliados pelas crianças numa escala de três pontos ( $3=$ sim, sempre; $2=$ mais ou menos; e 1=não, nunca). Para facilitar o preenchimento pelas crianças foram associadas a cada resposta três imagens de um smile, cada uma com uma cor diferente (1=verde; 2=amarelo; $3=$ vermelho) e utilizado um boneco do mesmo sexo do entrevistado representando um(a) aluno (a) que estaria prestes a transitar ou a iniciar o primeiro ano, dependendo da situação escolar da criança. Uma pontuação mais elevada indica uma melhor adaptação escolar.

School Social Behavior Scales - Social Competence Scale (SSBS-2; Merrell, 2002; adaptação portuguesa de Raimundo et al., 2012). Esta escala tem como objetivo avaliar a competência social de crianças e jovens entre os cinco e os 18 anos de idade e é composta por 32 itens organizados em três subescalas: A subescala Relações com Pares contém 14 itens que medem as habilidades sociais ou características que são importantes no estabelecimento de relações positivas com os pares e na aceitação social por estes (eg. "Oferece ajuda aos outros alunos $\mathrm{N}^{\circ} 45 \cdot \mathrm{Vol} .3 \cdot 17-31 \cdot 2017$ 
quando eles o necessitam"). A subescala de Autocontrolo contempla 10 itens que medem competências sociais relacionadas com a autocontenção, cooperação e cumprimento das exigências das regras da escola e expectativas (eg. "Responde de forma apropriada quando é corrigido pelos professores"). Por último, a subescala de Comportamento Académico é constituída por 8 itens relacionados com o desempenho competente e envolvimento nas tarefas académicas (eg. "Completa os trabalhos escolares sem necessidade de ser lembrado(a) disso"). A resposta aos itens é dada numa escala de cinco pontos (de 1=não exibe o comportamento em questão a $5=$ exibe muito frequentemente). As pontuações mais elevadas indicam melhores competências sociais. Os estudos têm demonstrado uma boa consistência interna da escala, fiabilidade teste-reteste, validade convergente e divergente com outras escalas de avaliação de comportamento, e capacidade de diferenciar grupos com caraterísticas diferentes (Merrell, 2001, 2002).

A adaptação portuguesa da escala de competência social apoia a estrutura multidimensional da competência social apresentada na escala original, ainda que a estrutura final original não tenha sido exatamente replicada. A estrutura encontrada, com menos sete itens pertencentes a duas mini escalas que foram retiradas do modelo, demonstrou melhor ajustamento do que o obtido pela estrutura original no estudo da adaptação portuguesa de Raimundo et al. (2012). As subescalas apresentaram correlações significativas semelhantes às encontradas com a versão original (Raimundo et al., 2008), uma consistência interna de bom a muito bom (Raimundo et al., 2012), e capacidade de discriminar crianças consideradas em risco das crianças não consideradas em risco (Gomes, 2008).

\section{Procedimento}

A recolha dos dados da versão para professores do Questionário de Adaptação Escolar e da escala de Competência Social do SSBS foi realizada individualmente pelos educadores e professores de quatro escolas e quatro jardins-deinfância da região de Lisboa que avaliaram todas as crianças a frequentar o último ano do préRevista Iberoamericana de Diagnóstico y Evaluación - e Avaliação Psicológica. RIDEP · N45 · Vol.3 · 17-31 2017 escolar e o primeiro ano de escolaridade das suas turmas. A versão para crianças do Questionário de Adaptação Escolar foi aplicada individualmente às crianças pela investigadora principal deste estudo em salas disponibilizadas para o efeito pelas escolas participantes. Estas amostras fizeram parte de um estudo de avaliação de eficácia de um programa de promoção de competências sócioemocionais na transição, sendo por isso amostras de conveniência. Para a realização do estudo foi efetuado previamente o pedido de autorização à direção dos agrupamentos de escola e aos diretores dos jardins-de-infância e escolas de primeiro ciclo envolvidos. Posteriormente foi obtido o consentimento informado escrito para participar no estudo por parte dos educadores, professores e pais de todas as crianças envolvidas. Este estudo foi submetido e aprovado pelo Conselho Científico da Faculdade de Psicologia da Universidade de Lisboa, a entidade responsável pela sua avaliação científica e ética na época.

\section{Análise Estatística}

A base de dados foi construída utilizando o programa estatístico SSPS (versão 21.0) através do qual realizámos a análise fatorial exploratória das duas versões do Questionário de Adaptação Escolar e analisámos a fidedignidade dos instrumentos e a sua validade concorrente. Para a sua análise fatorial confirmatória e da escala de Competência Social do SSBS-2 foi utilizado o software R (R Core Team, 2015), em particular o pacote lavaan (Rosseel, 2012).

Para analisar a estrutura fatorial das duas versões do Questionário de Adaptação Escolar foi efetuada uma análise fatorial exploratória através da análise dos componentes principais com rotação Varimax. No que se refere ainda à validade de constructo destes instrumentos foi também realizada a análise da correlação entre as subescalas e escalas do Questionário de Adaptação Escolar e as subescalas e a escala de Competência Social do SSBS-2 através do coeficiente de correlação de Pearson. A estrutura obtida foi depois validada através de análise fatorial confirmatória.

Para a análise da fidedignidade dos instrumentos foram utilizadas duas medidas: o coeficiente alfa de Cronbach para avaliar a consistência interna das diversas escalas e 
subescalas e o coeficiente de correlação de Pearson para avaliar a fidedignidade teste-reteste numa subamostra de professores $(\mathrm{N}=13)$ que reavaliaram 176 crianças com um intervalo temporal de sete meses. Foram ainda analisadas as diferenças em função do género e do nível escolar - pré-escolar e primeiro ano - utilizando o teste $t$ de Student.

A análise fatorial confirmatória (CFA) da escala de Competência Social do SSBS-2 foi realizada de acordo com os procedimentos efetuados nas análises da versão original (Crowley \& Merrell, 2003) e da adaptação portuguesa (Raimundo et al., 2012). Para ultrapassar as dificuldades da análise de dados baseada nos itens individuais foi seguida a sugestão de Collins e Gleaves (1998) de "análise indireta dos itens". Para cada subescala foram criadas mini escalas resultantes da combinação de dois a quatro itens, num total de 10 mini escalas (três a quatro por subescala). A análise da estrutura tri-fatorial do instrumento foi testada considerando o modelo exato da versão original e o modelo ajustado apresentado no estudo da versão portuguesa do instrumento (Raimundo et al., 2012).

Os modelos confirmatórios foram avaliados com base no método robusto MLM (quaselikelihood estimator) e os seguintes índices foram analisados para aferir a sua qualidade: Quiquadrado Santorra-Bentler( $\left.\mathrm{S}-\mathrm{B} \chi^{2}\right)$, Comparative Fit Index (CFI), Tucker-Lewis Index (TLI), Root Mean Square Error of Approximation (RMSEA) com 90\% intervalo de confiança, Standardized Root Mean Square Residual (SRMR). Os valores do CFI, GFI e TLI devem ser superiores a .90 (Bentler, 1990) e o RMSEA e SRMR inferior a .08 (Hu \& Bentler, 1999). Para verificar a invariância das estruturas obtidas, e assim comparar os modelos entre sexo e nível de escolaridade dos participantes, efetuaram-se análises multi-grupos impondo restrições de igualdade aos pesos fatoriais, médias e resíduos. Para análise da invariância verificou-se a significância da diferença do qui-quadrado face aos modelos testados (Maroco, Campos, Vinagre, $\&$ Pais-Ribeiro, 2014).

\section{Resultados}

Estrutura Fatorial e Fidedignidade das Versões para Professores e para Crianças do Questionário de Adaptação Escolar

Numa primeira análise fatorial exploratória da versão inicial do Questionário de Adaptação Escolar para professores foi retirado um item que revelava fraca comunalidade $(<0,4)$. Na segunda análise, o resultado do teste estatístico KaiserMeyer-Olkin (KMO) foi de .733, indicando que os dados eram adequados para a análise fatorial, da qual resultou uma estrutura com dois fatores que explicou $71,03 \%$ da variância: Adaptação Comportamental, com 3 itens associados ao ajustamento do comportamento às regras e rotinas do contexto escolar, e Motivação Escolar, com 4 itens indicando interesse e gosto pela escola e pela aprendizagem. O peso fatorial foi elevado para todos os itens e variou de .541 a .928 . A análise fatorial confirmatória deste instrumento revelou um ajustamento aos dados adequado $\left(\chi^{2}=42.605\right.$, $p=.000, \mathrm{CFI}=.96$, TLI $=.92$, RMSEA $=.10$, CI $90 \%=.07 \mid .12$, SRMR=.08). Os pesos fatoriais foram significativos, oscilando entre .46 e .91 (Figura 1), com confirmação de invariância parcial estrita entre nível de escolaridade e parcial escalar entre sexo (consultar Quadro 1).

O coeficiente alfa de Cronbach foi de .904 e de .718 para os dois fatores, e de .811 para a escala completa. A pontuação pode variar entre 3 e 15 na subescala de Adaptação Comportamental, e entre 4 e 20 na subescala Motivação Escolar, tendo variado no presente estudo entre 3 e 15 e entre 8 e 20 , respetivamente.

Da análise exploratória da estrutura fatorial da versão para crianças do Questionário de Adaptação Escolar (KMO de .870) resultou uma estrutura de fator único que explicou $41,36 \%$ da variância. $\mathrm{O}$ peso fatorial dos itens variou entre .533 e .764. Em termos confirmatórios, os índices revelaram um bom ajustamento do modelo testado $\left(\chi^{2}=35.069, \quad p=.137, \quad\right.$ CFI $=.97, \quad$ TLI $=.96$, RMSEA $=.03$, CI $90 \%=.00 \mid .05$, SRMR=.04), com todos os pesos fatoriais significativos entre .46 e .74 (Figura 2). A validade fatorial desta estrutura foi testada entre sexo e nível de escolaridade, tendo-se verificado invariância 
Quadro 1. Verificação da invariância entre nível de escolaridade e sexo

\begin{tabular}{lcccccccc}
\hline & \multicolumn{3}{c}{ Nível de Escolaridade } & \multicolumn{5}{c}{ Sexo } \\
\hline Modelos & S-B $\chi^{2}$ & df & BIC & df, S-B Diff $\chi^{2}$ & S-B $\chi^{2}$ & df & BIC & df, S-B Diff $\chi^{2}$ \\
QAEP & & & & & & & & \\
Modelo Configural & 88.554 & 22 & 4025.9 & - & 74.512 & 22 & 4063.3 & - \\
Invariância Métrica & 90.511 & 27 & 3999.2 & $5,1.6035^{\text {n.s }}$ & 76.377 & 27 & 4036.5 & $5,1.6320^{\text {n.s }}$ \\
Invariância Escalar & 97.098 & 32 & 3977.1 & $5,7.0327^{\text {n.s }}$ & 78.718 & 30 & 4021.6 & $3,2.4291^{\text {n.s }}$ \\
Invariância Estrita & 99.139 & 37 & 3950.5 & $5,1.3220^{\text {n.s1 }}$ & 107.886 & 39 & 3999.2 & $9,11.2318^{\text {n.s }}$ \\
QAEC & & & & & & & & \\
Modelo Configural & 126.26 & 54 & 4715.7 & - & 148.30 & 54 & 4915.5 & - \\
Invariância Métrica & 142.64 & 62 & 4686.2 & $8,7.3821^{\text {n.s }}$ & 153.44 & 62 & 4874.7 & $8,2.8171^{\text {n.s }}$ \\
Invariância Escalar & 150.40 & 70 & 4648.0 & $8,8.7341^{\text {n.s }}$ & 159.05 & 70 & 4834.3 & $8,5.9635^{\text {n.s }}$ \\
Invariância Estrita & 343.05 & 79 & 4788.9 & $9,192.65^{* * *}$ & 193.92 & 79 & 4817.5 & $9,13.6354^{\text {n.s }}$ \\
& & & & & & & & \\
SSBS & & & & & 71.920 & 28 & 9781.3 & - \\
Modelo Configural & 65.326 & 28 & 9492.9 & - & & & & \\
Invariância Métrica & 74.050 & 33 & 9698.9 & $5,9.0628^{\text {n.s }}$ & 73.276 & 33 & 9753.8 & $5,1.4468^{\text {n.s }}$ \\
Invariância Escalar & 78.383 & 37 & 9680.1 & $4,0151^{\text {n.s }}$ & 77.334 & 38 & 9729.0 & $5,0.5345^{\text {n.s }}$ \\
Invariância Estrita & 109.191 & 42 & 9682.1 & $5,16.5576^{* *}$ & 89.887 & 46 & 9695.4 & $8,0.3546^{\text {n.s }}$ \\
\hline
\end{tabular}

Nota: n.s = não significativo; n.s ${ }^{1}=$ invariância estrita parcial, itens 1 e 5 parâmetros livres;

n. $s^{2}=$ invariância escalar parcial, itens 8 e 17 parâmetros livres; *** $p<.000$; ** $p<.001$

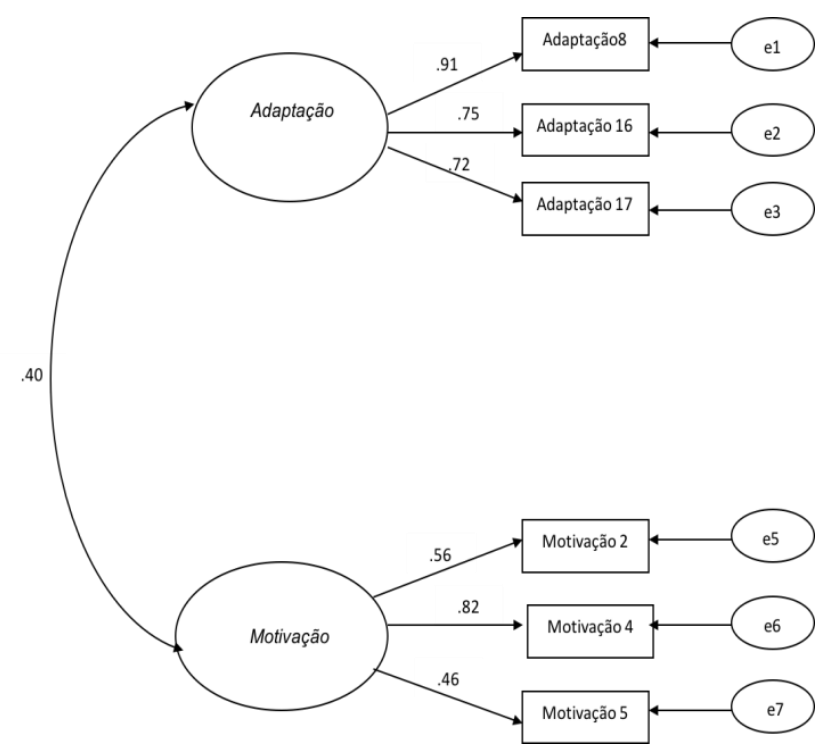

Figura 1. Estrutura confirmatória do Questionário de Adaptação Escolar para professores

escalar em termos de nível de escolaridade e invariância estrita ao nível do sexo (Quadro 1). O coeficiente alfa de Cronbach foi de .819. A pontuação pode variar entre 9 e 27, tendo no presente estudo variado entre 9 e 27.

Para uma subamostra $(\mathrm{N}=13$ educadores/professores e $\mathrm{N}=176$ alunos) foi realizado o reteste com um intervalo temporal de sete meses. As correlações entre o teste e o reteste dos fatores e da escala total do Questionário de Adaptação Escolar - versão para professores revelaram-se significativas $(p<.01)$ e elevadas
(Cohen, 1988), com coeficientes de correlação entre .583 e .673 . A correlação entre o teste e o reteste do Questionário de Adaptação Escolar versão para crianças revelou-se significativa, mas apenas moderada (Cohen, 1988) (Quadro 2).

Validade Fatorial e Fidedignidade da Escala de Competência Social do SSBS-2

A validade fatorial foi testada através da análise fatorial confirmatória do modelo proposto por Crowley e Merrell (2003) e do modelo final modificado na adaptação portuguesa de Raimundo et al. (2012). Com base nos resultados obtidos por Crowler e Merrell (2003) e Raimundo et al.(2012), o modelo testado na presente amostra obteve um ajustamento adequado aos dados $\chi^{2}=34.757, \quad p=.002, \quad$ CFI $=.99, \quad$ TLI $=.98$, RMSEA $=.07 \quad, \quad$ CI $90 \%=.04 \quad .09$, SRMR=.02).Todos os pesos fatoriais foram significativos oscilando entre .79 e .96 (consultar Figura 3) e verificou-se invariância escalar deste instrumento entre nível de escolaridade e estrita entre sexo (Quadro 1).

O valor do alfa de Cronbach no presente estudo foi de .949 para a subescala Relações entre Pares (três mini escalas), de .918 para o Autocontrolo (duas mini escalas), e de .943 para a Comportamento Académico (três mini escalas). Para a escala total o valor do alfa de Cronbach foi de .971 . As pontuações podem variar entre $11 \mathrm{e}$ 
Quadro 2. Correlação entre o Questionário de Adaptação Escolar, versões para professores e para crianças e as subescalas e escala de Competência Social do SSBS-2, e Coeficientes de correlação de Pearson entre o teste e reteste $(\mathrm{N}=176)$

\begin{tabular}{lccccc}
\hline & $\begin{array}{c}\text { Adaptação } \\
\text { (Crianças) }\end{array}$ & $\begin{array}{c}\text { Motivação } \\
\text { Escolar } \\
\text { (Professores) }\end{array}$ & $\begin{array}{c}\text { Adaptação } \\
\text { Comportamental } \\
\text { (Professores) }\end{array}$ & $\begin{array}{c}\text { Adaptação } \\
\text { global } \\
\text { (Professores) }\end{array}$ & $\begin{array}{c}r \\
\text { teste- } \\
\text { reteste }\end{array}$ \\
\hline $\begin{array}{l}\text { Comportamento } \\
\text { Académico }\end{array}$ & $.182^{* *}$ & $.514^{* *}$ & $.842^{* *}$ & $.830^{* *}$ & $\mathbf{. 6 9 0}^{* *}$ \\
$\begin{array}{l}\text { Autogestão } \\
\text { Relação entre }\end{array}$ & .052 & $.451^{* *}$ & $.893^{* *}$ & $.829^{* *}$ & $\mathbf{. 7 1 2}$ \\
pares & .108 & $.533^{* *}$ & $.712^{* *}$ & $.754^{* *}$ & $\mathbf{. 6 6 9 * *}$ \\
$\begin{array}{l}\text { Escala global } \\
\text { SSBS }\end{array}$ & $.129^{*}$ & $.545^{* *}$ & $.867^{* *}$ & $.863^{* *}$ & $\mathbf{. 7 0 0 * *}$ \\
$\begin{array}{l}\text { Adaptação } \\
\text { (Crianças) }\end{array}$ & & $.182^{* *}$ & .101 & $.165^{* *}$ & \\
$r$ teste-reteste & $\mathbf{. 3 4 9 * *}$ & $\mathbf{. 5 8 3 * *}$ & $\mathbf{. 6 7 3}^{* *}$ & $\mathbf{. 6 6 5}^{* *}$ & \\
\hline$p<.01$ & & & & &
\end{tabular}

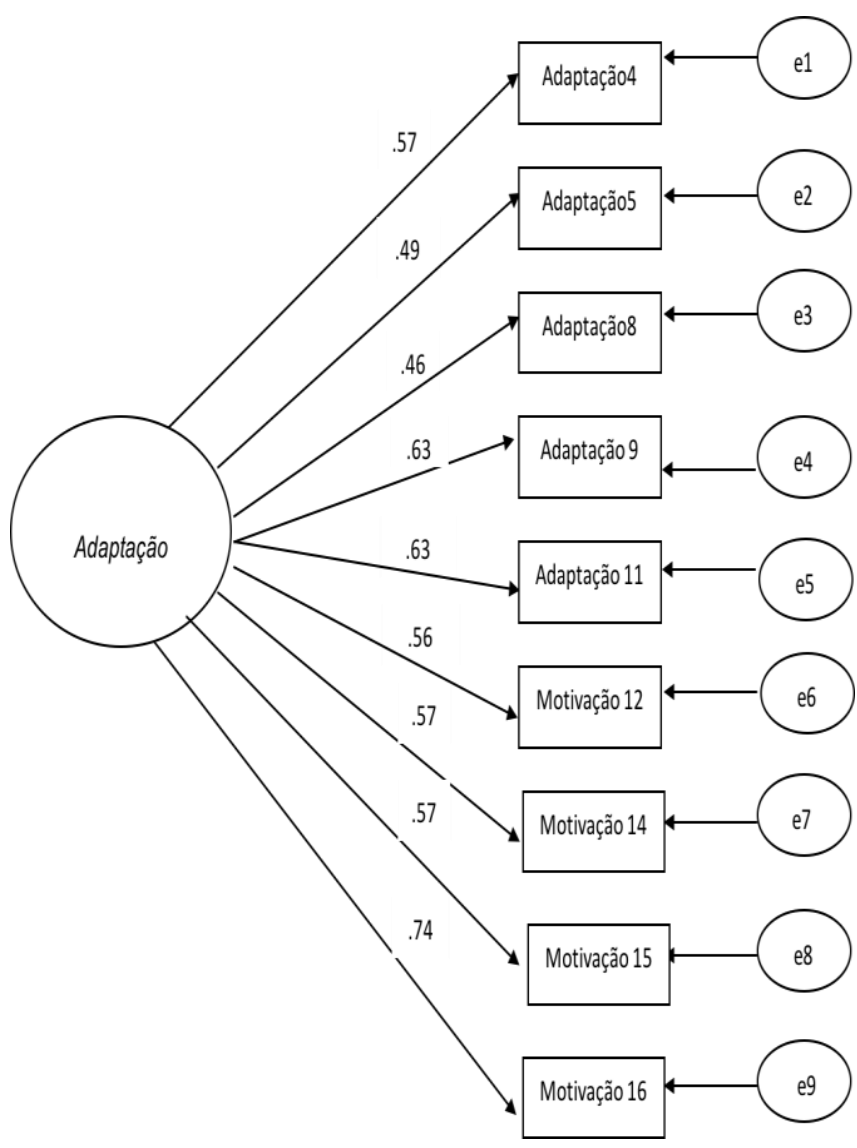

Figura 2. Estrutura confirmatória do Questionário de Adaptação Escolar para crianças

55 na primeira subescala, entre 7 e 35 na segunda subescala, e entre 8 e 40 na terceira subescala, tendo no presente estudo variado entre 12 e 55 , entre 10 e 35 e entre 8 e 35 , respetivamente.

Para uma subamostra $(\mathrm{N}=13$ professores e $\mathrm{N}=176$ alunos) foi realizado o reteste com um intervalo temporal de sete meses. As correlações entre o teste e reteste foram significativas $(p<.01)$ e elevadas (Cohen, 1988) com coeficientes de correlação entre .669 e .712 (Quadro 2).

Correlações entre e Escala de Competência Social do SSBS-2 e as duas Versões do Questionário de Adaptação Escolar

Para testar a validade concorrente das duas versões do Questionário de Adaptação Escolar foram primeiramente analisadas as correlações entre as subescalas e escala de Competência Social do SSBS-2 e as subescalas e o índice global de Adaptação Escolar - versão para professores, através do coeficiente de correlação de Pearson. Todas as correlações foram, de acordo com o esperado, positivas, estatisticamente significativas $(p<.01)$, e de moderadas a elevadas (Cohen, 1988), com coeficientes de correlação entre .451 e .893 (Quadro 2). O Questionário de Adaptação Escolar - versão para crianças revelou uma correlação positiva e significativa $(p<.01)$, embora fraca, apenas com a subescala de Comportamento Académico e com a escala de Competência Social do SSBS-2.

Finalmente, a versão para crianças do Questionário de Adaptação Escolar revelou uma correlação significativa, embora fraca, com a subescala de Motivação Escolar, e com o índice global da versão para professores deste questionário $(p<.01)$.

Estatística Descritiva dos Valores do Questionário de Adaptação Escolar e da Escala de Competência Social do SSBS-2 e Diferenças de Média em Função do Género e do Nível Escolar 
Quadro 3. Valores médios no Questionário de Adaptação Escolar, versões para professores e para crianças, e na Escala de Competência Social do SSBS-2, e diferenças de médias em função do nível de escolaridade

\begin{tabular}{lcccc}
\hline & TOTAL & PRẼ & $1^{\circ}$ ANO & \\
& $\mathrm{M}(\mathrm{DP})$ & $\mathrm{M}(\mathrm{DP})$ & $\mathrm{M}(\mathrm{DP})$ & $t$ \\
\hline Adaptação Escolar (Professores) & $30.27(3.87)$ & $30.66(3.79)$ & $29.67(3.93)$ & $2.275^{*}$ \\
Motivação Escolar & $18.19(2.07)$ & $18.12(2.16)$ & $18.29(1.94)$ & -.763 \\
Adaptação Comportamental & $12.08(2.54)$ & $12.55(2.39)$ & $11.37(2.62)$ & $4.105^{* *}$ \\
Adaptação Escolar (Crianças) & $24.19(3.62)$ & $23.51(3.85)$ & $25.25(2.97)$ & $-4.501^{* *}$ \\
Escala de Competência Social & $92.19(19.66)$ & $96.72(19.17)$ & $85.00(18.76)$ & $5.388^{* * *}$ \\
Comportamento Académico & $30.22(7.34)$ & $27.67(6.17)$ & $24.31(6.43)$ & $4.744^{* *}$ \\
Autocontrolo & $26.56(5.44)$ & $27.42(5.45)$ & $25.14(5.28)$ & $3.715^{* *}$ \\
Relação entre pares & $35.40(8.44)$ & $41.53(8.72)$ & $35.80(8.83)$ & $5.773^{* *}$ \\
\hline${ }^{*}<<.05 ;{ }^{* *} p<.01$ & & & &
\end{tabular}

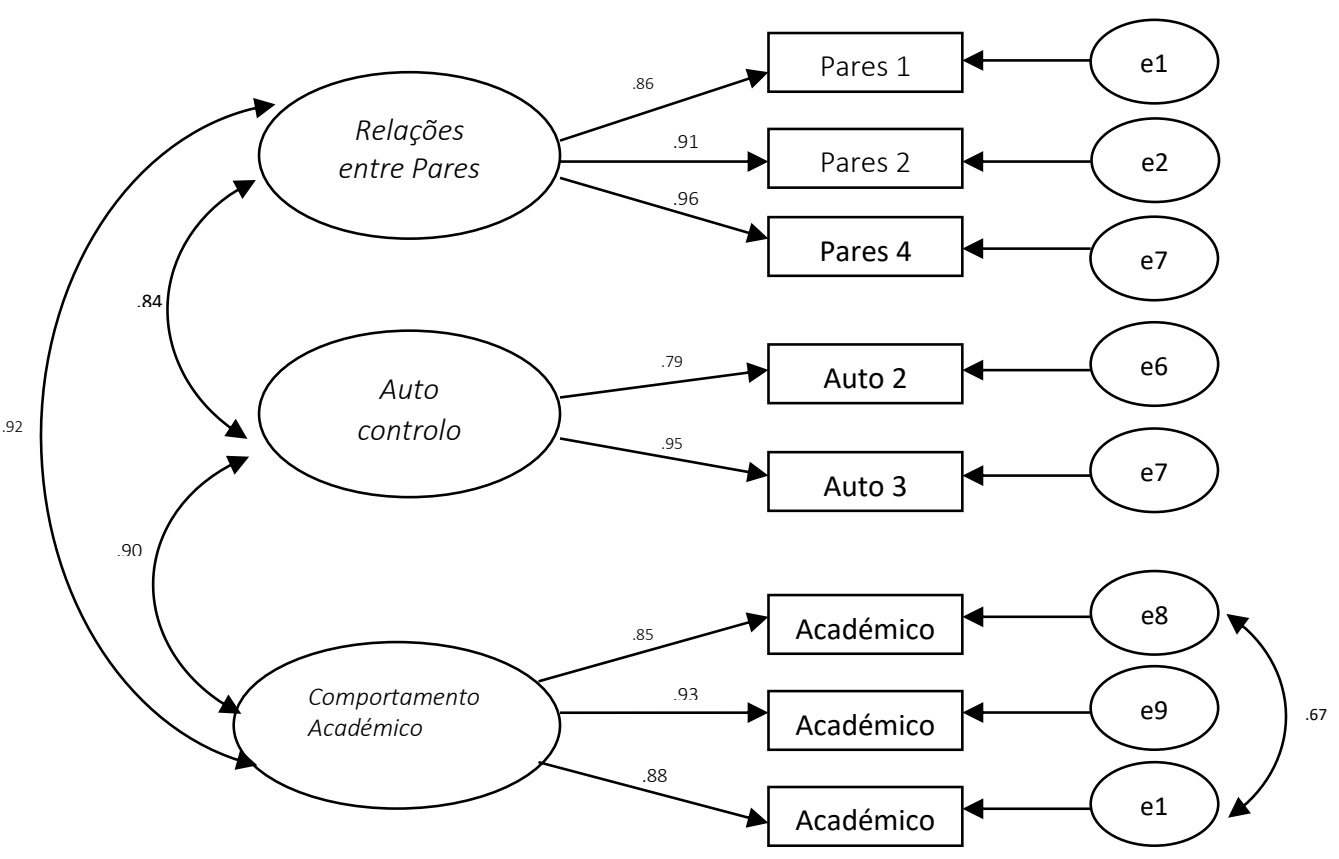

Figura 3. Modelo final da Escala de Competência Social do SSBS-2, de Raimundo et al. (2012)

Os valores médios obtidos no total da amostra na adaptação escolar avaliada por professores e crianças e na escala de Competência Social do SSBS-2 são apresentados no Quadro 3.

Não se verificaram diferenças significativas em função do género quanto à Motivação Escolar $(\mathrm{t}=.438 ; \mathrm{N}=328 ; p>.05)$, e aos índices globais da Adaptação Escolar avaliada pelos professores $(\mathrm{t}=1.793 ; \mathrm{N}=328 ; p>.05)$ e pelas próprias crianças $(\mathrm{t}=.806 ; \quad \mathrm{N}=328 ; \quad p>.05)$. Verificou-se uma diferença significativa na Adaptação Comportamental $\quad(\mathrm{t}=2.376 ; \quad \mathrm{N}=328 ; \quad p<.05)$, avaliada pelos professores, com melhores resultados para as crianças de sexo feminino.
No que refere à escala de Competência Social do SSBS-2, foram encontradas diferenças significativas em função do género nas subescalas de Comportamento Académico $(\mathrm{t}=2.282 ; \mathrm{N}=328$; $p<.05)$ e de Autocontrolo ( $\mathrm{t}=2.683 ; \mathrm{N}=328$; $p<.01$ ), com melhores resultados para o sexo feminino. Verificou-se uma diferença próxima do nível de significância na escala de Competência Social total ( $\mathrm{t}=1.970 ; \mathrm{N}=328 ; \mathrm{p}=.05)$, e não foi encontrada diferença significativa na Relação entre Pares ( $t=1.090 ; \mathrm{N}=328 ; \mathrm{p}>.05)$.

Verificaram-se diferenças significativas de acordo com o nível escolar na Adaptação Comportamental $(\mathrm{t}=4.105 ; \mathrm{N}=328 ; p<.01)$, e nos 
índice globais da Adaptação Escolar avaliada pelos professores $(\mathrm{t}=2.275 ; \mathrm{N}=328 ; \quad p<.05)$, e pelas crianças $(\mathrm{t}=-4.501 \mathrm{~N}=328 ; \quad p<.01)$. As crianças a frequentar o último ano do pré-escolar revelaram melhor adaptação escolar global e melhor adaptação comportamental avaliada pelos professores do que as crianças do primeiro ano de escolaridade, e uma pior adaptação escolar avaliada pelos próprios. Não se verificou uma diferença significativa em função do nível escolar na Motivação Escolar ( $\mathrm{t}=-.763 ; \mathrm{N}=328 ; p>.05)$. No que refere à escala de Competência Social do SSBS-2, foram encontradas diferenças significativas em função do nível escolar nas subescalas Relação entre Pares ( $\mathrm{t}=5.773 ; \mathrm{N}=328$; $p<.01)$, Autocontrolo ( $\mathrm{t}=3.715 ; \mathrm{N}=328 ; p<.01) \mathrm{e}$ Comportamento Académico $(\mathrm{t}=4.744 ; \mathrm{N}=328$; $p<.01$ ), e na escala total de Competência Social $(\mathrm{t}=5.388 ; \mathrm{N}=328 ; p<.01)$. As crianças a frequentar o último ano do pré-escolar revelaram melhores competências de relacionamento com os pares, de autocontrolo e comportamento académico, e melhores competências sociais do que as crianças de primeiro ano de escolaridade (Quadro 3).

\section{Discussão}

O presente estudo teve por objetivo construir um Questionário de Adaptação Escolar, com uma versão de heteroavaliação pelos professores e outra de autoavaliação pelas crianças, e analisar alguns dos seus parâmetros de validade e fiabilidade bem como da escala de Competência Social do School Social Behavior Scales (SSBS-2; Merrell, 2002; adaptação portuguesa de Raimundo et al., 2012).

A análise da versão para professores do Questionário de Adaptação Escolar revela uma estrutura em dois fatores que traduzem componentes diferentes, um associado à motivação escolar, e outro associado à capacidade de adaptar o comportamento face às novas expectativas. Ambos os fatores e a escala total revelaram bons índices de consistência interna e boa precisão e estabilidade temporal. Considerando a variedade de definições de adaptação escolar no início da escolaridade formal encontradas na literatura (eg. McIntyre, Blacher e Baker, 2006; Serdal, 2010) e os domínios nos quais a adaptação é entendida (Perry \& Weinstein, 1998), podemos considerar que o questionário de adaptação escolar para professores avalia as dimensões Comportamental e Motivacional, sendo necessário desenvolver futuramente outros instrumentos que contemplem a adaptação do ponto de vista social e académico para ter uma visão mais ampla.

Apesar da versão para crianças do Questionário de Adaptação Escolar revelar uma estrutura de fator único com um bom índice de consistência interna, a correlação entre o teste e o reteste com um intervalo de sete meses, numa subamostra, foi significativa mas mais baixa do que a encontrada com a versão para professores. A tarefa de solicitar autorrelatos confiáveis e válidos a crianças pequenas é dificultada por uma série de fatores, nomeadamente os seus limites cognitivos, níveis inconsistentes de envolvimento com a entrevista ou autorrelato, e os efeitos de resposta e vieses motivacionais que tendem a distorcer o relato das crianças em relação às suas caraterísticas (Byrne, 1996). As pesquisas com base em medidas de autorrelato com crianças sugerem que estas têm tendência para responder com base no seu estado no momento da avaliação e não de acordo com níveis mais gerais de ajustamento, podendo ser medidas menos confiáveis (Deighton et al, 2014). Também existem indícios de um menor relato de dificuldades comportamentais em crianças pequenas (eg. Edelbrock, Costello, Dulcan, Kalas, \& Conover, 1985).

As correlações encontradas entre as duas subescalas e a escala total da versão para professores do Questionário de Adaptação Escolar e as subescalas e escala de Competência Social foram todas significativas sugerindo que os dois fatores encontrados e o índice global de Adaptação Escolar avaliada por professores medem aspetos do comportamento adaptativo. No que refere a versão para crianças do Questionário de Adaptação Escolar foram encontradas correlações significativas, embora fracas, com a subescala de Comportamento Académico e a escala de Competência Social do SSBS-2. Se analisarmos o conteúdo dos itens verificamos que a versão para crianças do Questionário de Adaptação Escolar contempla, sobretudo itens relacionados com o comportamento académico, o 
que justifica a relação significativa encontrada. Foram ainda encontradas correlações significativas fracas entre a versão para crianças do Questionário de Adaptação Escolar e a subescala de Motivação Escolar e escala total da versão para professores do mesmo questionário. Enquanto a versão para professores implica uma heteroavaliação efetuada por estes, o questionário de adaptação para crianças resulta da perceção que estas têm em relação ao seu comportamento, com as limitações já acima referidas (eg. Byrne, 1996).

Em termos gerais, os resultados sugerem que o Questionário de Adaptação Escolar para professores pode ser considerado um instrumento válido para avaliar a dimensão comportamental e motivacional da adaptação escolar das crianças na transição para o primeiro ano. Tendo sido construído com base nas perceções de educadores e professores no contexto português, os itens são de fácil compreensão, e com validade ecológica. O Questionário de Adaptação Escolar para crianças demonstrou uma boa consistência interna mas um índice de estabilidade temporal mais fraco, pelo que a leitura dos resultados com base neste instrumento deverá ser efetuada com mais cautela. Através destes questionários pretende-se caraterizar o grupo e fornecer pistas de intervenção de forma a facilitar o processo de transição.

A avaliação que os educadores e professores fizeram da adaptação das crianças e a avaliação efetuada pelas próprias é globalmente positiva. A análise das diferenças em função do nível de escolaridade indica diferenças significativas na Adaptação Escolar, com melhores índices de Adaptação Global e Adaptação Comportamental avaliadas pelo professor nas crianças finalistas do pré-escolar comparativamente às do $1^{\circ}$ ciclo. Estes resultados poderão estar relacionados com as exigências da transição para a escola, a qual tem sido percebida como um dos maiores desafios com que a criança se confronta nos primeiros anos da infância (Margetts, 2002). A entrada na escola implica um reajustamento às novas exigências intelectuais, sócio-emocionais e comportamentais da sala de aula do novo contexto escolar durante um período que é simultaneamente de grande mudança a nível do desenvolvimento (Perry \& Weinstein, 1998). As diferenças entre o préescolar, onde a organização e o horário são mais Revista Iberoamericana de Diagnóstico y Evaluación - e Avaliação Psicológica. RIDEP · No45 · Vol.3 · 17-31 2017 flexíveis, permitindo uma maior capacidade de decisão da criança, e a abordagem do primeiro ciclo mais dirigida e estruturada (Einarsdottir, 2006), implicam uma mudança nas expectativas em relação ao comportamento da criança. Assim, é expectável que a avaliação do comportamento da criança pelo educador no pré-escolar seja mais favorável, face à avaliação efetuada pelo professor no início do primeiro ano, uma vez ainda não existir nesta fase o conhecimento e domínio das regras e expectativas comportamentais do novo contexto. Apesar do desafio inerente à transição, os níveis de Motivação Escolar parecem manterse após a mesma. Os estudos demonstram que as crianças encaram a transição para a escola como um acontecimento positivo associado a um sentimento de promoção (eg. Correia \& MarquesPinto, 2017; Dockett \& Perry, 2012). Não foram encontradas diferenças significativas na adaptação escolar em função de género o que sugere que nesta faixa etária o processo de adaptação é semelhante em rapazes e raparigas.

No que refere à escala de Competência Social do SSBS-2, os resultados apoiam a estrutura multifatorial apresentada nos estudos da escala original e da versão portuguesa do SSBS-2 (Crowley \& Merrell, 2003; Merrell, 2002; Raimundo et al., 2012). A estrutura encontrada no estudo da adaptação portuguesa do instrumento revelou-se mais ajustada à amostra do presente estudo do que a da escala original.

Relativamente à fidedignidade da escala, os resultados demonstram uma elevada consistência interna tanto para as mini escalas como para a escala total, tal como no estudo de Raimundo et al. (2012). A análise da correlação entre o teste e o reteste com um intervalo de sete meses de uma subamostra do estudo sugere uma boa precisão e estabilidade temporal, já obtida no estudo da escala (Merrell, 2002).

No geral estes resultados sugerem que a escala de Competência Social do SSBS-2 se adequa à avaliação das competências sociais de crianças no final do pré-escolar e no primeiro ano de escolaridade.

Foram encontradas diferenças significativas em função do género, com melhores resultados no Comportamento Académico e Autocontrolo nas raparigas, tal como seria de esperar face aos resultados obtidos na escala original (Merrell, 
2002) e em estudos com a versão portuguesa (Gomes, 2008; Raimundo et al., 2012). Ao contrário dos resultados não significativos obtidos nos estudos de Raimundo et al (2012) e de Merrell (2002), no presente estudo as crianças finalistas do pré-escolar obtiveram melhores resultados do que as crianças de primeiro ano de escolaridade tanto nas subescalas como na escala de Competência Social. No estudo de Major e Seabra-Santos (2004b), também se verificou uma redução das aptidões sociais avaliadas pelos educadores, dos 5 para os 6 anos de idade, justificada pelo facto de metade da amostra de 6 anos de idade estar a frequentar a escolaridade básica com outras exigências face ao jardim-deinfância. Tendo sido a avaliação, no presente estudo, efetuada no primeiro trimestre do ano escolar, e considerando as mudanças inerentes à transição para o primeiro ciclo anteriormente referidas, podemos considerar que exista alguma vulnerabilidade do ponto de vista social associada à necessidade de reajustar o comportamento às novas regras e de construir novas relações sociais.

\section{Limitações e implicações para o futuro}

Apesar dos resultados promissores, algumas limitações devem ser apontadas ao presente estudo. As amostras foram recolhidas por conveniência não sendo representativas da população portuguesa, tendo cada professor/educador avaliado várias crianças, o que poderá ter determinado alguns enviesamentos nos resultados. De futuro, será importante realizar novos estudos de análise da fidedignidade e validade dos questionários de adaptação escolar com amostras mais alargadas da população. Por outro lado, os dados resultaram da avaliação subjetiva feita por professores / e por crianças, sendo necessário em estudos futuros complementar esta informação com a avaliação por outros informantes (e.g. pais) e a observação direta da criança. Em estudos futuros será ainda importante realizar análises multinível de modo a distinguir a variância a nível do grupo da variância individual.

De todo o modo, este estudo apresenta uma análise preliminar de qualidades psicométricas de dois instrumentos de medida relevantes para a avaliação da adaptação à transição escolar sugerindo que, em particular a versão para Revista Iberoamericana de Diagnóstico y Evaluación-e Avaliaço Psicologica. professores do Questionário de Adaptação e a escala de Competência Social da SSBS, são instrumentos promissores para a avaliação da adaptação escolar e da competência social das crianças na transição para a escola.

\section{Referências}

Akcinar, B. (2013). The predictors of school adaptation in early childhood. Procedia Social and Behavioral Sciences, 93, 10991104.

Alexander, K. L., Entwisle, D. R., \& Dauber, S. L. (1993). First-grade classroom behavior: Its short- and long-term consequences for school performance. Child Development, 64, 801814.

Baydar, N., Küntay, A., Gökşen, F., Yağmurlu, B., \& Cemalc1lar, Z. (2007). Adaptive social behavior inventory. Unpublished manuscript.

Bentler, P. M. (1990). Comparative fit indexes in structural models. Psychological Bulletin, 107, 238-246.

Birch, S. H., \& Ladd, G. W. (1997). The teacherchild relationship and children's early school adjustment. Journal of School Psychology, 35, 61-79.

Byrne, B. (1996). Measuring self-concept across the life span: Issues and instrumentation. In Measurement and instrumentation in psychology, (pp. 69-84). Washington, DC, US: American Psychological Association.

Cassidy, J., \& Asher, S. R. (1992). Loneliness and peer relations in young children. Child Development, 63, 350-365.

Cohen, J. (1988). Statistical power analysis for the behavioral sciences (2nd Ed.). New Jersey: Lawrence Erlbaum.

Collaborative for Academic, Social, and Emotional Learning (2003). Safe and sound: An educational leader's guide to evidencebased social and emotional learning (SEL) programs. Retrieved from CASEL website: http://casel.org/publications/safe-and-soundan-educational-leaders-guide-to-evidencebased-sel-programs/

Collins, J., \& Gleaves, D. (1998). Race, job applicants, and the five-factor model of personality: Implications for black psychology, industrial/ organizational 
psychology, and the five-factor theory. Journal of Applied Psychology, 83, 531-544.

Consortium on the school-based promotion of social competence (1996). The school-based promotion of social competence: Theory, research, practice, and policy. In R. J. Haggerty, L. R. Sherrod, N. Garmezy \& M. Rutter (Eds.), Stress, risk, and resilience in children and adolescents: Processes, mechanisms, and interventions (pp. 268316). New York, NY: Cambridge University Press.

Correia, K., \& Marques-Pinto, A. (2016). Adaptation in the transition to school: Perspective of parents, pre-school and primary school teachers. Educational Research, 58, 247-264

Correia, K., \& Marques-Pinto, A. (2017). Transición a la escuela primaria: Escuchando la voz de los niños [Transition to primary school: hearing children's voices]. (manuscript submitted for publication).

Crowley, S., \& Merrell, K. (2003). The structure of the School Social Behavior Scales: A confirmatory factor analysis. Assessment for Effective Intervention, 28, 41-55.

Deighton, J., Croudace, T., Fonagy, P., Brown, J., Patalay, P., \& Wolpert, M. (2014). Measuring mental health and wellbeing outcomes for children and adolescents to inform practice and policy: A review of child self-report measures. Child and Adolescent Psychiatry and Mental Health, 8:14.

Denham, S. A. (1986). Social cognition, prosocial behavior, and emotion in preschoolers: Contextual validation. Child Development, 57, 194-201.

Denham, S. A. (2006). Social-emotional competence as support for school readiness: What is it and how do we assess it? Early Education and Development, 7, 57-89.

Dockett, S., \& Perry, B. (2012). "In kindy you don't get taught": Continuity and change as children start school. Frontiers of Education in China, 7, 5-32.

Edelbrock, C., Costello, A. J., Dulcan, M.K., Kalas, R., \& Conover, N. C. (1985). Age differences in the reliability of the psychiatric interview of the child. Child Development, 56, 265-275.
Einarsdottir, J. (2006). From pre-school to primary school: When different contexts meet. Scandinavian Journal of Educational Research, 50, 165-184.

Elias, J. E., \& Weissberg, M. J. (2000). Social and emotional learning: Promoting the development of all students. Journal of Educational and Psychological Consultation, 17, 233-255.

Gomes, E. (2008). Validação da versão portuguesa de uma escala de competências sociais para crianças [Validation of the portuguese version of one social competence scale for children]. Dissertação de Mestrado não publicada [Unpublished master's thesis], ISCTE - Lisbon University Institute, Lisbon, Portugal.

Gresham, F. M., \& Elliott, S. N. (1990). Social skills rating system. Circle Pines. MN: American Guidance Service.

Hamre, B., \& Pianta, R. (2001). Early teacherchild relationships and the trajectory of children's school outcomes through eighth grade. Child Development, 722, 625-638.

Hemmeter, M. L., Ostrosky, M., \& Fox, L. (2006). Social and emotional foundations for early leaming: A conceptual model for intervention. School Psychology Review, 35, 583-601.

Holopainen, N., Lappalainen, K., Junttila, N., \& Savolainen, H. (2012). The role of social competence in the psychological well-being of adolescents in secondary education. Scandinavian Journal of Educational Research, 56, 199-212.

Hu, L., \& Bentler, P. (1999). Cutoff criteria for fit indexes in covariance structure analysis: Conventional criteria versus new alternatives. Structural Equation Modeling: A Multidisciplinary Journal, 6(1), 1-55.

Ladd, G. W., \& Price, J. M. (1987). Predicting children's social and school adjustment following: The transition from preschool to kindergarten. Child Development, 58, 11681189.

Lane, K. L., Menzies, H. M., Barton-Arwood, S. M., Doukas G. L., \& Munton, S. M. (2005). Designing, implementing, and evaluating social skills interventions for elementary students: Step-by-step procedures based on Vol.3 $\cdot 17-31 \cdot 2017$ 
actual school-based investigations. Preventing School Failure: Alternative Education for Children and Youth, 49, 18-26.

Lemos, M., \& Meneses, H. (2002). A avaliação da competência social: Versão portuguesa da forma para professores do SSRS [Social competence assessment: The portuguese version of SSRS teacher scale]. Psicologia: Teoria e Pesquisa, 18, 267-274.

Machado, P. M., Veríssimo, M., \& Denham, S. (2012). O teste de conhecimento de emoções para crianças de idade pré-escolar. Revista Iberoamericana de Diagnóstico y Evaluación - e Avaliação Psicológica, 34(2), 201-222.

Major, S. (2011). Avaliação de aptidões sociais e problemas de comportamento em idade préescolar: Retrato das crianças portuguesas [Assessment of social skills and problem behaviors in preschoolers: A picture of Portuguese children] (Doctoral dissertation, Universidade de Coimbra, Coimbra, Portugal). Retrieved from https://estudogeral .sib.uc.pt/bitstream/10316/17774/5/Tese_Sofi a\%20Major.pdf

Major, S., \& Seabra-Santos, M. (2014a). Factor validation of the Portuguese version of the social skills scale of the preschool and kindergarten behavior scales. Paidéia, 24, 145-153.

Major, S., \& Seabra-Santos, M. (2014b). Aptidões e problemas de comportamento: Retratos das crianças portuguesas em idade pré-escolar. Revista Iberoamericana de Diagnóstico y Evaluación - e Avaliação Psicológica, 30(2), 69-92.

Margetts, K. (2002, October). Starting school matters: Supporting children's transition to school. Paper presented at the Early Childhood Matters Conference, Melbourne.

Margetts, K. (2009). Early transition and adjustment and children's adjustment after six years of schooling. European Early Childhood Education Research Journal, 17, 309-324.

Maroco, J. Campos, J., Vinagre, M., \& PaisRibeiro, J. (2013). Adaptação transcultural Brasil-Portugal da escala de satisfação com o suporte social para estudantes do ensino superior. Psychology/Psicologia, Reflexão e Crítica, 27(2), 247-256.
McIntyre, L. L., Blacher, J., \& Baker, B. L. (2006). The transition to school: Adaptation in young children with and without intellectual disability. Journal of Intellectual Disability Research, 50, 349-361.

Merrell, K. (2001). Assessment of children's social skills: Recent developments, best practices, and new directions. Exceptionality, 9, 3-18.

Merrell, K. (2002). School Social Behavior Scales, Second Edition. Eugene, OR: Assessment-Intervention Resources.

Nurss, J. R., \& McGauvran, M. E. (1986). Metropolitan Readiness Test. New York: Harcourt Brace Jovanovich.

Pedro, C., \& Albuquerque, C. (2007). Questionário de avaliação de aptidões sociais: SSRS - versão alunos [Social skills assessment questionnaire: SSRS - student version]. Psychologica, 45, 87-102.

Perry, K. E., \& Weinstein, R. S. (1998). The social context of early schooling and children's school adjustment. Educational Psychologist, 33, 177-194.

Raimundo, R., Carapito, E., Pereira, A. I., Marques-Pinto, A., Lima, L,. \& Ribeiro, T. (2012). School social behavior scales (SSBS2): An adaptation study of the Portuguese version of the social competence scale. Spanish Journal of Psychology, 15, 14731484.

Rosseel, Y. (2012). Lavaan: An R Package for Structural Equation Modeling. Journal of Statistical Software, 48(2), 1-36. URL http://www.jstatsoft.org/v48/i02/

Serdal, S. (2010) Attachment and social behaviours in the period of transition from preschool to first grade. Social Behavior \& Personality: An International Journal, 38, 347-357.

Silva, R., Veríssimo, M., \& Santos, A. (2004). Adaptação psicosocial da criança ao préescolar. Análise Psicológica, 22, 109-118.

Strayer, F., \& Noël, J. M. (1990). Adaptation psychosociale de l'enfant en milieu préscolaire, questionnaire APSE. Montréal: Documento inédito.

Zins, J., \& Elias, M. (2006). Social and emotional learning. In G. Bears \& K. Minke (Eds.). Children's needs III: Development, 
prevention, and intervention (pp.1-13).

Bethesda, MD: National Association of

School Psychologists. 